\title{
Granular Parakeratosis
}

\author{
Maren Gaul, DO; Jonathan Bass, MD; Schield Wikas, DO
}

\section{PRACTICE POINTS}

- Granular parakeratosis most commonly presents in middle-aged women in the axillae.

- The cause is unknown but possibly related to irritation from rubbing, occlusion, sweating, or deodorants.

- Multiple treatment modalities likely contribute to clearing, the most important being removal of any triggering topical products.

To the Editor:

A 46-year-old overweight woman presented with a rash in the axillae of 2 months' duration. She did not report any additional symptoms such as pruritus or pain. She reported changing her deodorant recently from Secret Original to Secret Clinical Strength (both Procter \& Gamble). Her medical history was remarkable for asthma and gastroesophageal reflux disease. Clinical examination revealed erythematous-brown, stuccolike, hyperkeratotic papules coalescing into plaques in recently shaved axillae, affecting the left axilla more than the right axilla (Figure 1). The clinical differential diagnosis included granular parakeratosis, intertrigo, Hailey-Hailey disease, Darier disease, pemphigus vegetans, confluent and reticulated papillomatosis, acanthosis nigricans, seborrheic keratoses, and irritant or allergic contact dermatitis. A punch biopsy revealed a marked compact parakeratotic horn with retention of keratohyalin granules (Figure 2). The subjacent epidermis showed some acanthosis and spongiosis with mild chronic inflammation of the dermal rim. Based on histopathology, granular parakeratosis was diagnosed.

At a subsequent visit 2 weeks later, we prescribed glycolic acid lotion $10 \%$ applied to the axillae twice daily, plus tretinoin gel $0.05 \%$ applied to the axillae each evening. She reported clearing after 1 week of therapy. She also had changed her deodorant from Secret Clinical Strength back to the usual Secret Original. The patient discontinued topical treatment after clearing of the lesions. Three weeks later, clinical examination revealed postinflammatory hyperpigmentation in the axillae, and the prior lesions had resolved (Figure 3).

Granular parakeratosis is an unusual condition most commonly presenting in middle-aged women in the axillae, with a clinical presentation of erythematous to brownish hyperkeratotic papules coalescing into plaques. Although few cases have been reported, granular parakeratosis likely is more common than has been reported. There have been reports involving the scalp, cheeks, abdomen, thighs, and other intertriginous areas including inguinal folds and the submammary region. ${ }^{1-4}$ There also is an infantile form related to diapers and zinc oxide paste. ${ }^{5}$ Although uncommon, granular parakeratosis can occur as a single papule or plaque and is termed

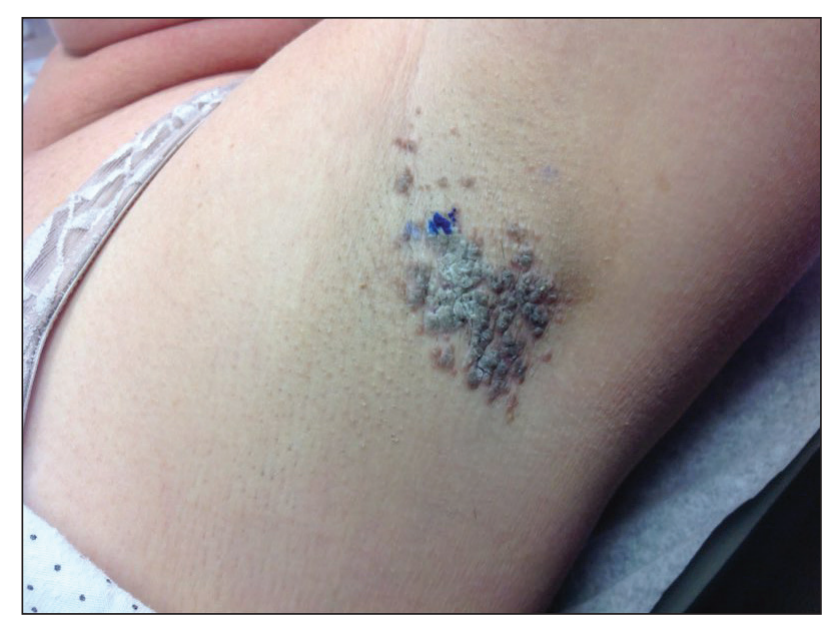

FIGURE 1. Left axilla on initial presentation with erythematous-brown, stuccolike, hyperkeratotic papules coalescing into plaques.

Dr. Gaul was from Western Reserve Hospital, Cuyahoga Falls, Ohio, and currently is from Tanana Valley Clinic, Fairbanks, Alaska. Dr. Bass is from the School of Medicine, Case Western Reserve University, Beachwood, Ohio. Dr. Wikas is from Tri-County Dermatology, Cuyahoga Falls.

The authors report no conflict of interest.

Correspondence: Maren Gaul, DO, 1001 Noble St, Ste 300, Fairbanks, AK 99701 (marenness@gmail.com). 


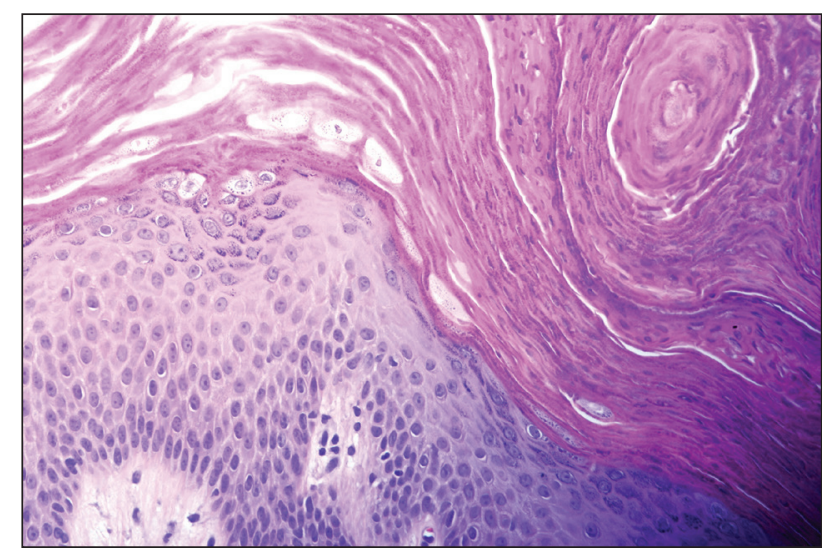

FIGURE 2. Marked compact parakeratotic horn with retention of keratohyalin granules $(\mathrm{H \& E}$, original magnification $\times 40)$.

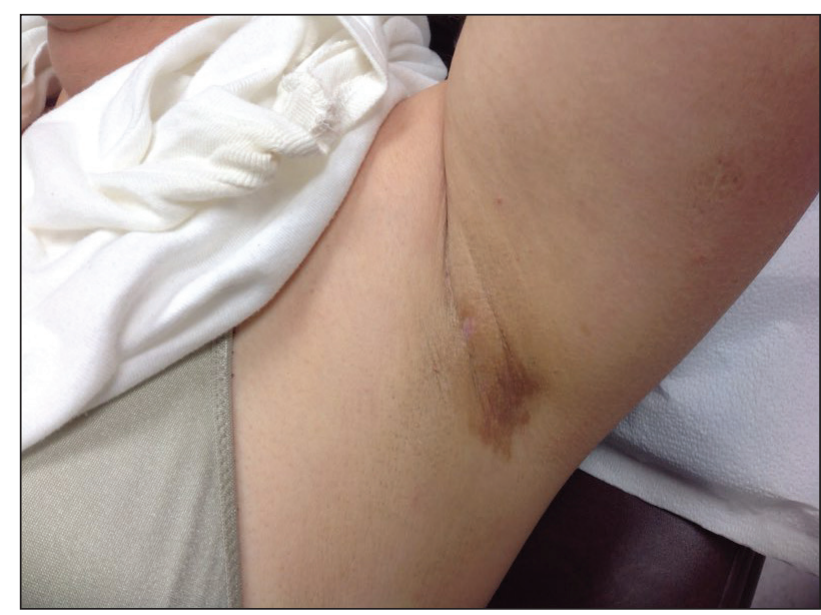

FIGURE 3. Left axilla following treatment with glycolic acid lotion $10 \%$ and tretinoin gel $0.05 \%$.

granular parakeratotic acanthoma. ${ }^{6}$ Lesions may persist for months, spontaneously resolve and recur, and occasionally evolve into fissures and erosions due to irritation. Pruritus is a common concern. Histology of granular parakeratosis reveals hyperkeratosis with eosinophilic staining, compact parakeratosis with retention of basophilic keratohyalin granules, and vascular proliferation and ectasia. ${ }^{5}$

The cause is unknown but possibly related to irritation from rubbing, occlusion, sweating, or deodorants. ${ }^{5,7}$ Cases indicate a link to obesity. Hypotheses as to the etiology include the disruption of cornification. Normally, filaggrin maintains the keratohyaline granules in the stratum corneum during cornification. Therefore, the retention of keratohyaline granules in granular parakeratosis may be due to a defect in processing profilaggrin to filaggrin, which has been proposed based on ultrastructural and immunohistochemical studies. ${ }^{8}$
The differential diagnosis includes granular parakeratosis, intertrigo (caused by seborrheic dermatitis, candidiasis, inverse psoriasis, or erythrasma), Hailey-Hailey disease, Darier disease, pemphigus vegetans, confluent and reticulated papillomatosis, and irritant or allergic contact dermatitis. The papules may resemble seborrheic keratoses, while the plaques can be mistaken for acanthosis nigricans.

Therapeutic success has been reported with topical corticosteroids, vitamin D analogues, topical or oral retinoids, ammonium lactate, calcineurin inhibitors, topical or oral antifungals, cryotherapy, and botulinum toxin injections. ${ }^{3,9-11}$ In addition, parakeratosis has decreased in biopsies from psoriatic patients after acitretin, methotrexate, and phototherapy, which may be alternative treatments for unusually difficult or recalcitrant cases of granular parakeratosis. To minimize side effects and resolve the papules quickly, we combined 2 synergistic agents-glycolic acid and tretinoin-each with different mechanisms of action, and we observed excellent clinical response.

Granular parakeratosis is possibly related to a combination of topical products that potentiate irritation, rubbing, and occlusion of sweat. Multiple treatment modalities likely contribute to clearing, the most important being removal of any triggering topical products. Our patient's change in deodorant may have been the inciting factor for the disease. Withdrawal of the Secret Clinical Strength deodorant prompted clearing, though topical retinoid and glycolic acid acted as facilitating therapies for timely results. A thorough history, as highlighted by this case, may help pinpoint etiologic factors. By identifying a seemingly innocuous change in hygienic routine, we were able to minimize the need for ongoing therapy.

\section{REFERENCES}

1. Graham R. Intertriginous granular parakeratosis: a case report and review of the literature. J Am Acad Dermatol. 2011;64:AB45-AB45.

2. Compton $A K$, Jackson JM. Isotretinoin as a treatment for axillary granular parakeratosis. Cutis. 2007;80:55-56.

3. Channual J, Fife DJ, Wu JJ. Axillary granular parakeratosis. Cutis. 2013;92;61, 65-66.

4. Streams S, Gottwald L, Zaher A, et al. Granular parakeratosis of the scalp: a case report. J Am Acad Dermatol. 2007;56:AB81-AB81.

5. James WD, Berger T, Elston D. Andrews' Diseases of the Skin. 12th ed. Philadelphia, PA: Elsevier, Inc; 2015.

6. Resnik KS, Kantor GR, DiLeonardo M. Granular parakeratotic acanthoma. Am J Dermatopathol. 2005;27:393-396.

7. Naylor E, Wartman D, Telang G, et al. Granular parakeratosis secondary to postsurgical occlusion. J Am Acad Dermatol. 2008;58:AB126.

8. Bolognia JL, Jorizzo JL, Schaffer JV. Dermatology. 3rd ed. Philadelphia, PA: Elsevier, Inc; 2012.

9. Baum B, Skopit S. Granular parakeratosis treatment with tacrolimus $0.1 \%$ ointment: a case presentation and discussion. J Am Osteo Coll Dermatol. 2013;26:40-41.

10. Brown SK, Heilman ER. Granular parakeratosis: resolution with topical tretinoin. J Am Acad Dermatol. 2002;47:S279-S280.

11. Webster CG, Resnik KS, Webster GF. Axillary granular parakeratosis: response to isotretinoin. J Am Acad Dermatol. 1997;37:789790. 